\title{
CÍRCULO VICIOSO Y REVOLUCIÓN CRÍTICA EN LA TEORÍA DE LOS PROBLEMAS DE GILLES DELEUZE: EL ROL DEL ÁLGEBRA EN DIFERENCIA Y REPETICIÓN*
}

\section{VICIOUS CIRCLE AND CRITICAL REVOLUTION IN GILLES DELEUZE'S THEORY OF PROBLEMS: THE ROLE OF ALGEBRA IN DIFFERENCE AND REPETITION}

\author{
Gonzalo Santaya \\ https://orcid.org/0000-0002-1998-6215 \\ gonsantaya@gmail.com \\ Consejo Nacional de Investigaciones Cientificas y Técnicas \\ Universidad de Buenos Aires, Argentina
}

RESUMEN Este artículo busca contribuir a la elucidación del concepto de "problema" elaborado por Gilles Deleuze en Diferencia y repetición, analizando la presencia en dicho concepto del algebrista noruego Niels Henrik Abel. Deleuze atribuye a este matemático el haber creado un método que rompe con el "círculo vicioso" que consiste en calcar los problemas a imagen y semejanza de sus soluciones. Argumentaremos que esta interpretación de Deleuze se apoya en la obra de Jules Vuillemin La philosophie de l'algèbre, donde se traza un paralelo entre el álgebra de Lagrange y la filosofía trascendental de Fichte

* Article submitted on 24/06/2020. Accepted on 10/12/2020. 
para mostrar ese vicio común a sus métodos. Comenzaremos por reconstruir el mencionado "círculo vicioso", para luego recorrer las características generales del problema algebraico y sus paralelismos con la filosofía crítica, señalando finalmente los puntos principales que Deleuze busca rescatar del método abeliano -presentado por Vuillemin como la superación de Lagrange (y, por extensión, de Fichte).

Palabras clave Deleuze. Problema. Vuillemin. Álgebra. Filosofía crítica.

ABSTRACT This paper aims to shed light on the concept of "problem" developed by Gilles Deleuze in Difference and repetition by analyzing the presence in that book of the Norwegian algebraist Niels Henrik Abel. Deleuze attributes to this mathematician having invented a method that breaks the "vicious circle" which consists in tracing the problems to the image and similarity of the solutions. We will argue that Deleuze's interpretation is based on Jules Vuillemin's work La philosophie de l'algèbre, which draws a parallelism between Lagrange's algebra and Fichte's transcendental philosophy to show a common flaw in both their methods. We will begin by characterizing the aforementioned "vicious circle", and then go through the general characteristics of the algebraic problem and its parallel with critical philosophy, in order to show, finally, the main features that Deleuze seeks to highlight in the abelian method-presented by Vuillemin as the overcoming of Lagrange (and, consequently, of Fichte).

Keywords Deleuze. Problem. Vuillemin. Algebra. Critical philosophy.

\section{Introducción}

Uno de los ejes principales de Diferencia y repetición es el de desarrollar una teoría de los problemas, entendidos no solo como estadios psicológicos, pedagógicos o epistemológicos, sino como instancias virtuales, a la vez ontológicas y trascendentales, momentos fundamentales de la producción de lo real y de la experiencia efectiva. El célebre capítulo III de esa obra, "La imagen del pensamiento", presenta una serie de dispositivos - Deleuze habla allí de "postulados"- que obstruyen el acceso del pensamiento a esa dimensión problemática y productiva. ${ }^{1}$ Si bien el objetivo de Deleuze es específicamente

1 La cuestión de la imagen del pensamiento no es solo un momento central de Diferencia y repetición, sino una de las principales preocupaciones que atraviesa la obra deleuziana en su conjunto. Para un recorrido general de esta cuestión puede verse Canavera (2015). 
filosófico, su tipología de postulados reenvía también a otras disciplinas y dominios donde ellos operan. Una de las disciplinas predilectas de Deleuze a la hora de extraer ejemplos y herramientas para su propia filosofía es la matemática: Diferencia y repetición abunda en referencias a nociones del análisis matemático, la geometría diferencial y el álgebra, para determinar esta teoría de los problemas. En el presente artículo nos detendremos en algunas referencias algebraicas de las que Deleuze se sirve al exponer dicha teoría. Mostraremos que esta apropiación deleuziana sigue las tesis expuestas por Jules Vuillemin en La philosophie de l'algèbre (1962) - principalmente en su lectura filosófica de los aportes de Niels Henrik Abel (Noruega, 1802-1829) a la teoría de las ecuaciones algebraicas. ${ }^{2}$

La compleja ontología desarrollada en Diferencia y repetición se despliega en los dos planos de lo actual y lo virtual, o solución y problema, planos que se encuentran en una situación de presuposición mutua y de correspondencia sin semejanza. El capítulo IV de esa obra cifra en el cálculo diferencial - y sus interpretaciones metafísicas - el movimiento general de los problemas: la relación diferencial aparece allí como la 'mitad virtual', y las funciones integrales determinadas por ella como la 'mitad actual' del objeto matemático. Sin embargo, Deleuze pretende ampliar esta exposición más allá del cálculo, pues lo virtual determina todos los dominios de lo real (tanto de lo real empírico como de lo real matemático): "Ni siquiera podemos considerar que, técnicamente, el cálculo diferencial sea la única expresión matemática de los problemas en tanto tales. [...] Más recientemente, este rol ha podido ser mejor cumplido por otros procedimientos" (Deleuze, 1968, p. 232-233). ${ }^{3}$ Deleuze se refiere aquí a los algebristas Abel y Galois. ${ }^{4}$

Concretamente, al analizar el teorema de Abel sobre la irresolubilidad de ecuaciones algebraicas de quinto grado o superior, Vuillemin desarrolla

2 Jules Vuillemin (1920-2001), filósofo francés que trabajó en un amplio espectro de problemas y temáticas, y con quien Deleuze tuvo cierto contacto - por mediación de Foucault - para ocupar un puesto que aquel dejaría en la Universidad de Clermont-Ferrand (Cf. Dosse, 2007, p. 365). Muchas de sus obras son ocasionalmente citadas por Deleuze, y en particular La philosophie de l'algèbre. Ella aparece en Deleuze (1968, p. 234), Deleuze y Guattari (1980, p. 603), y Deleuze (2006, p. 72), en referencia - respectivamente - al método de Abel, a la noción de multiplicidad de Riemann, y al método aritmético-estructural de Weierstrass. Esta diversidad de temas y referencias da cuenta de la gran influencia de la obra de Vuillemin en cuanto a la relación de Deleuze con la matemática.

3 En todos los casos (tanto para la obra de Deleuze como de Vuillemin) seguimos la paginación de la edición original francesa indicada en 'Bibliografía', y la traducción es propia.

4 Hemos analizado en detalle la cuestión del cálculo diferencial en Deleuze en Santaya (2017). Sobre la relación Deleuze-Galois, puede verse Santaya (2014) y Abadi (2015). La presencia de Abel, en cambio, ha sido muy poco trabajada. Ella es mencionada superficialmente por Duffy (Cf. 2013, pp. 84-88), quien hace de la recepción deleuziana de Abel y Galois un mero subcapítulo de la teoría de los diferenciales en su lectura de la Idea deleuziana, sin detenerse en los aportes específicos que Deleuze rescata. Por otra parte, la notable influencia de Vuillemin en esta referencia ha sido ampliamente pasada por alto en la bibliografía especializada. 
una serie de reflexiones fundamentales para la concepción deleuziana de los problemas como instancias virtuales que gozan de un modo de existencia heterogéneo y genético con respecto a sus soluciones. Deleuze encuentra en Abel un procedimiento que rompe con el 'círculo vicioso' en la teoría de los problemas virtuales, el cual consiste en enfocar a los mismos siguiendo la imagen de sus soluciones. Si bien el procedimiento abeliano pertenece por entero al dominio del álgebra, Vuillemin lo inserta en un contexto en el cual el método matemático y el método filosófico corren a la par y resuenan en sus distintos momentos. Sin embargo - como suele suceder en las referencias deleuzianas - la mención a Abel es breve y oscura. Reconstruir el sentido de esta mención permitirá mostrar cómo, por un lado, el círculo vicioso acecha en distintos campos del pensamiento, y cómo, por otro lado, este círculo puede ser superado mediante un método crítico que habilita el acceso al plano virtualproblemático.

En lo que sigue, atravesaremos cinco momentos con el fin de abordar la teoría deleuziana de los problemas bajo la luz de su referencia a Abel. En primer lugar (2), expondremos la naturaleza del círculo vicioso que impide la captación de los problemas como tales, a través de las 'ilusiones' propias de la imagen dogmática del pensamiento. A continuación (3), reconstruiremos el contexto del problema de las ecuaciones algebraicas, siguiendo los aportes de Lagrange y de sus predecesores, y mostrando cómo ellos caen en dichas ilusiones. Luego (4), desarrollaremos las implicancias filosóficas que Vuillemin extrae de Lagrange, al relacionar su método con el de Fichte (en un primer paralelismo entre matemática y filosofía trascendental). Esto permitirá mostrar la afinidad del 'círculo vicioso' deleuziano con los análisis algebraico-filosóficos de Vuillemin. En cuarto lugar (5), desarrollaremos el abordaje de Abel a las ecuaciones algebraicas, señalando las razones por las que este rompe - según Vuillemin - con el vicio metodológico de Lagrange (y, por extensión, con el 'círculo vicioso'). Finalmente (6), explicitamos las implicancias del método de Abel en la teoría deleuziana de los problemas virtuales (segundo paralelismo matemática-filosofía trascendental), enfatizando porqué este matemático realiza una revolución crítica "más considerable que la copernicana" (Deleuze, 1968, p. 234).

\section{Círculo vicioso e ilusiones dogmáticas}

¿Qué significa atribuir a los problemas un carácter virtual? Significa atribuirles una potencia interna por la cual ellos generan sus soluciones - ya sea esta solución un dominio científico, ya un objeto o estado de cosas empírico-, de 
las cuales se dirá que actualizan esa potencia. Un problema, así entendido, posee una existencia paradójica, no enunciable discursivamente ni experimentable en la intuición espacio-temporal. El riesgo de llamar 'problema' a esta instancia virtual - y 'solución' a su contracara actual - es considerarlo como una mera proposición interrogativa, con su correlativa respuesta 'ya hecha' esperando ser descubierta, lo cual elimina ese potencial genético. Al considerar el problema como una mera proposición interrogativa que decanta en una proposición afirmativa (o negativa), ponemos ambas instancias como pertenecientes a un mismo plano de existencia, un mismo régimen de enunciación, afectadas por diferentes coeficientes enunciativos: interrogación y afirmación (o negación). En tanto pensamos el problema desde los medios simbólicos establecidos por los dominios de resolución o de enunciación preexistentes, caemos en un círculo vicioso que bloquea el acceso a un auténtico pensamiento del problema en tanto virtual. Deleuze expresa este círculo del siguiente modo: "un problema no es resoluble sino en la medida en que es 'verdadero', pero tenemos tendencia a definir la verdad de un problema por su resolubilidad" (Deleuze, 1968, p. 233). A causa de esta 'tendencia', la potencia virtual interna del problema es sofocada por el criterio externo de las soluciones actuales constituidas: al definir la resolubilidad mediante la verdad, traficamos implícitamente la forma particular de una verdad ya establecida, que se considera isomorfa al problema. El pensamiento, atrapado en este círculo, no puede avanzar hacia la génesis de nuevas verdades. La apuesta deleuziana es dar a la forma del problema un carácter tal que exceda y funde lo que hay de verdadero en sus soluciones. ${ }^{5}$

El círculo vicioso es descripto con más detalle en el Capítulo III de Diferencia y repetición, a partir de la distinción de dos tipos de ilusión: la natural y la filosófica. Según la ilusión natural (Cf. Deleuze, 1968, pp. 203-204), los problemas son directamente calcados de las proposiciones que les sirven de solución. Por ejemplo, si pregunto "¿es el hombre 'animal racional'?”, o “existe Dios?”, esto no plantea propiamente un problema, sino que transforma en interrogación una proposición cuyas significaciones y designaciones se pretenden como dadas de antemano con un sentido unívoco. La respuesta a una pregunta semejante apela entonces a una forma de correspondencia entre palabras y cosas preexistentes, que permitirían determinar inequívocamente la

5 En este sentido, consideramos desacertada la explicación de Simon Duffy (2013, p. 84), quien propone: "En lugar de basar el criterio extrínseco de resolubilidad, i. e., la verdad, sobre el carácter interno del problema como Idea, Deleuze propone hacer a este criterio dependiente del 'simple criterio exterior', i. e., de las condiciones mismas del problema como tal." (La traducción es propia.) Consideramos, y así lo intentaremos argumentar en lo que sigue, que las "condiciones del problema como tal" son el "criterio interno del problema", y que es el "criterio extrínseco de resolubilidad" el que "depende" de ellas. 
verdad o falsedad de la proposición. De este modo, el mundo de la representación se cierra sobre sí mismo, encubriendo la instancia problemática y extraproposicional que - siguiendo la ontología deleuziana - subyace a la génesis de los significados de las proposiciones y de los estados de cosas designados por ellas, articulando su correspondencia recíproca en la experiencia.

La ilusión filosófica (Cf. Deleuze, 1968, pp. 207-209) es más compleja, pues pretende poner a las proposiciones en contacto con problemas, elevando las categorías de 'verdad' y 'falsedad' al análisis de los problemas mismos, y no ya de las proposiciones. El filósofo se pregunta ahora por las condiciones de posibilidad de afirmar con verdad que "Dios existe", o que "el hombre es animal racional". La cuestión se traslada entonces a las condiciones formales de las proposiciones, para evaluar la naturaleza de los problemas (verdadero o falso) de acuerdo a ellas. Se intenta desarrollar así un medio para discriminar los problemas según su posibilidad de recibir una solución, explicitando reglas bajo las cuales una solución es posible y válida a priori. El falso problema sería aquél que no es resoluble apelando a estas condiciones formales supuestas como único medio válido, necesario y universal, para la expresión de soluciones. Pero en la medida en que estas condiciones formales remitan a la forma de las soluciones, y no a la del problema, este continúa siendo subordinado a condiciones externas, y lo trascendental se concibe aún a imagen y semejanza de lo empírico.

Ambos tipos de ilusión convierten al problema en una instancia subsidiaria de las soluciones actuales (ya en su particularidad empírica, ya en su generalidad abstracta), en lugar de pensar lo problemático como un ámbito con una lógica propia, independiente de derecho del contenido y la forma de sus soluciones, y tal que su propia estructura interna determine las condiciones bajo las cuales esas soluciones son posibles. Esto ocurre en los distintos campos del pensamiento:

Encontramos siempre los dos aspectos de la ilusión: la ilusión natural que consiste en calcar los problemas sobre las proposiciones que se suponen preexistentes, opiniones lógicas, teoremas geométricos, ecuaciones algebraicas, hipótesis físicas, juicios trascendentales; y la ilusión filosófica, que consiste en evaluar los problemas según su 'resolubilidad', es decir según la forma extrínseca variable de la posibilidad de su solución (Deleuze, 1968, pp. 209-210; cursivas mías).

La ilusión filosófica anima el círculo vicioso por el cual el sabio, devenido agente reproductor de la imagen dogmática del pensamiento, pretende elevarse al análisis de los problemas, pero reenvía su verdad a la forma de las soluciones. 
Deleuze denuncia este vicio en Aristóteles, Descartes, Kant, ${ }^{6}$ por mencionar a algunos. Pero no deja de recordar que ambas ilusiones acechan en otros dominios, como en la historia de la matemática:

Más precisamente, desde un punto de vista geométrico y sintético, los problemas son inferidos de proposiciones de un tipo particular que llamamos teoremas. Es una tendencia de la geometría griega, por un lado, limitar los problemas en beneficio de los teoremas, por otro lado subordinar los problemas a los teoremas mismos. [...] Desde un punto de vista algebraico y analítico, lo esencial de la situación no cambia. Los problemas son ahora calcados sobre ecuaciones algebraicas, y evaluados según la posibilidad de efectuar sobre los coeficientes de la ecuación un conjunto de operaciones que provea las raíces. Pero, al igual que en geometría imaginamos el problema resuelto, en álgebra operamos sobre cantidades desconocidas como si fueran conocidas: así se prosigue el trabajo que consiste en reducir los problemas a la forma de las proposiciones capaces de servirles de caso de solución (Deleuze, 1968, p. 208). ${ }^{7}$

Tanto la geometría como el álgebra han perpetuado en sus métodos la confusión entre la instancia virtual-problemática y la instancia actualresolutiva: confunden, de este modo, lo empírico con lo trascendental. Lo que la filosofía deleuziana busca es una aproximación inmanente por la cual los problemas generen las condiciones de su resolución; pero en la medida en que consideramos a los problemas exclusivamente a partir de sus soluciones, nos mantenemos en un criterio meramente empírico, extrínseco o general-abstracto. En el caso del álgebra, las 'proposiciones' en cuestión son las ecuaciones, como enunciación matemática particular que pone una equivalencia $(=)$ entre dos miembros. Como veremos en lo que sigue, el planteo de los problemas algebraicos se cifró largamente en la consideración exclusiva de este tipo de proposiciones particulares, y en sus modos de conexión con otras proposiciones formalmente semejantes, sin llegar a vislumbrar las condiciones 'extraproposicionales' que generaban la posibilidad de dichos modos de conexión. Esa instancia extraproposicional, auténtico ámbito trascendental del pensamiento, es ciertamente difícil de aprehender: ella solo puede vislumbrarse a partir de los términos actuales que la expresan (soluciones), pero estos términos

6 Respecto a la concepción kantiana de los problemas, Deleuze escribe: "Kant define aún la verdad de un problema por su posibilidad de recibir una solución: se trata esta vez de una forma de posibilidad trascendental, conformemente a un uso legítimo de las facultades tal como es determinado en cada caso por tal o cual organización del sentido común (con la cual el problema corresponde). - Encontramos siempre los dos aspectos de la ilusión" (Deleuze, 1968, p. 209). Se trata del extrinsequismo o empirismo de Kant, denunciado por Deleuze no solo en su teoría de los problemas, sino también en su teoría de las facultades y en la de las Ideas dialécticas. Veremos cómo Fichte (en la lectura de Vuillemin) acaba incurriendo en el mismo problema. De la mano del método abeliano, Deleuze intentará eliminar este vicio del método trascendental.

7 Todo este pasaje prefigura lo que, más tarde, Deleuze y Guattari (Cf. 1980, pp. 440-464) conceptualizarán como la oposición entre una "ciencia mayor" y una "menor". 
proyectan sobre ella sus propiedades, transformando al productor en una imagen distorsionada por el producto. ${ }^{8}$

Como señalamos previamente, nos dedicaremos en lo que sigue al modo en que la historia del álgebra ha roto con este círculo vicioso. Pues, de hecho, "si un círculo tal ha sido roto, ello se debe principalmente al matemático Abel; es él quien elabora un método siguiendo el cual la resolubilidad debe desprenderse de la forma del problema [...]. Hay ahí una inversión radical de la relación solución-problema, una revolución más considerable que la copernicana" (Deleuze, 1968, p. 233, cursivas mías). ¿Cuál es ese método de Abel?, ¿en qué consiste su inversión y su revolución, y cómo se vincula con la heterogeneidad y relación intrínseca de lo virtual respecto a lo actual en la teoría de los problemas?

\section{Círculo vicioso en el álgebra: los matemáticos "precríticos" y el aporte de Lagrange}

Para comprender la novedad del planteo de Abel, es preciso reconstruir el contexto de las cuestiones que aborda. En la presente sección veremos brevemente en qué consiste el problema de las ecuaciones quínticas - o, más precisamente, de la forma de resolución general de polinomios de quinto grado o superior - que da lugar a su perspectiva innovadora. Comencemos aclarando los términos. Un polinomio general de grado $n$ es una función de la forma:

$$
P(x)=a x^{n}+b x^{n-1}+c x^{n-2}+\cdots+d x+e
$$

Es decir, una suma finita de términos que contienen una misma variable ( $x$ ) elevada a diferentes potencias, donde $a, b, c, d$, son los coeficientes, es decir, cantidades constantes expresables algebraicamente (por medio de sumas, restas, multiplicaciones, divisiones), $e$ es un término independiente (no ligado a la variable $x$ ), y $n$ es un número entero que indica el grado de la ecuación (la máxima potencia a la que está elevada la variable $x$ ). Obviamente, una infinidad de funciones individuales con estas características son concebibles, pues $n$ puede tomar cualquier valor entero, y los coeficientes, cualquier valor real.

Un problema asociado a estas funciones es el del cálculo de sus raíces, es decir, aquellos valores de $x$ para los cuales la expresión toma el valor cero.

8 En este sentido, Deleuze sigue a Albert Lautman (2011), que constituye una fuente esencial para comprender la teoría deleuziana de los problemas dialécticos en particular, y la presencia de la matemática en su pensamiento en general. Al respecto, ver Kretschel (2014) y Duffy (2013, pp. 117-135). 
De acuerdo con el teorema fundamental del álgebra, un polinomio de grado $n$ posee $n$ raíces. Es decir que para la ecuación general indicada arriba debe existir un conjunto A que contenga sus $n$ raíces:

$$
A=\left\{x_{1}, x_{2}, x_{3}, \ldots, x_{n-1}, x_{n}\right\}
$$

$x_{1}, x_{2}, x_{3}, \ldots$ son diferentes valores que, asignados a $x$, vuelven 0 a la función. Uno de los problemas principales de la historia del álgebra fue la determinación de fórmulas de resolución general, correspondientes a ecuaciones de distintos grados, construidas exclusivamente a partir de sus coeficientes; esto es, una fórmula tal que, usando los coeficientes de un polinomio de un grado determinado $n$, provea las $n$ raíces del mismo. Dicha fórmula lleva el nombre de "resolvente general"; ella se conoce, presumiblemente desde la antigua Babilonia, para la ecuación general de grado dos:

$$
a x^{2}+b x+c=0
$$

cuya resolvente general es:

$$
x=\frac{-b \pm \sqrt{b^{2}-4 a c}}{2 a}
$$

Cualesquiera sean los coeficientes $a, b$ y $c$, podemos reemplazarlos en esta fórmula general para obtener los dos valores de $x$ en los que la ecuación cuadrática propuesta valga 0 . El interés en hallar fórmulas generales para las raíces de polinomios de grados superiores a 2 se enciende en el renacimiento, especialmente luego de que el matemático italiano Scipione dal Ferro encontrara en el año 1500 una resolvente general para polinomios cúbicos de la forma $a x^{3}+b x-c=0$ (es decir, anulando el coeficiente del término $x^{2}$ ). Ferro no publica sus resultados porque en la época era común desafiar a otros matemáticos a resolver ecuaciones, y este secreto podía serle de utilidad para futuros duelos; pero los rumores acerca de la fórmula secreta no tardan en propagarse en el ambiente. El hallazgo es sospechado, buscado y retomado por otros matemáticos italianos como Tartaglia (el "tartamudo"), Cardano y su discípulo Ferrari, en una historia donde se mezclan la confianza, la traición, el celo por mantener el secreto y el avance en los descubrimientos. Cardano publica un tratado sobre la cuestión (ante la indignación de Tartaglia, que le había confiado la fórmula a condición de no publicarla). Otros matemáticos célebres como Viète, Descartes, Newton, Leibniz, Girard, Euler, Gregory, Harriot y Tschirnhaus avanzaron - 
durante los siglos XVI y XVII - en la búsqueda y perfeccionamiento de técnicas para las ecuaciones generales de tercer y cuarto grado. ${ }^{9}$ Ya durante el siglo XVIII se contaba con métodos de resolución general para los polinomios de segundo, tercer y cuarto grado. La búsqueda de la fórmula para el polinomio de quinto grado era entonces un tema recurrente. El problema era que las fórmulas resolventes (y sus demostraciones correspondientes) se volvían más y más engorrosas cuanto más aumentaba el grado de la ecuación propuesta, y los intentos de dar con la fórmula general para la ecuación de grado 5 (o superiores) parecían condenados al fracaso.

Lagrange fue uno de los matemáticos que contribuyó a encauzar la cuestión. $\mathrm{Su}$ intervención nos interesa especialmente porque la lectura comparativa que Vuillemin hace entre su planteo y el de Abel es fundamental para entender la revolución que, según Deleuze, este último realiza en la teoría de los problemas. En efecto, Vuillemin dedica largas páginas a desarrollar y contrastar los métodos de los algebristas prelagrangianos, de Lagrange y de Abel, páginas decisivas para comprender la crítica de Deleuze a las ilusiones dogmáticas vistas en la sección precedente, que afectaban al álgebra y la filosofía por igual. En términos de las "ilusiones" desarrolladas en la sección anterior, los algebristas prelagrangianos operaban buscando fórmulas que satisficieran la ecuación general ("¿es esta la fórmula resolvente general de la ecuación de grado cinco?”, “¿o esta otra?”, se preguntaban, condicionados por la ilusión natural); Lagrange, por su parte, analizará el problema en términos de sus condiciones formales (presa de la ilusión filosófica, preguntará: “¿bajo qué condiciones esta fórmula funciona como resolvente general?"). En ambos casos los planteos se sostienen sobre la consideración de ecuaciones particulares, incapaces de elevarse a un planteo general del problema (cosa que hará Abel, demostrando definitivamente la imposibilidad de una resolución general para ecuaciones de quinto grado o superior). Sin embargo, el método de Lagrange cuenta por sí mismo con un interés matemático y filosófico que prefigura la vía abeliana: este célebre matemático francés anuncia, según Vuillemin (1962, p. 73), el "método crítico en el Álgebra”, aunque será Abel quien llevará la razón algebraica a su auténtica y definitiva revolución crítica.

Lagrange aborda el problema, en un artículo de $1771,{ }^{10}$ mediante un análisis a la vez histórico (que consiste en un balance de los trabajos ya realizados en

9 Para un recorrido histórico preciso y una exposición de la publicación de Cardano y un resumen de los esfuerzos de los modernos en esta área, ver Kline (1972, pp. 351-361, y pp. 790-800).

10 Se trata de "Réflexions sur la résolution algébrique des équations", analizado en Vuillemin (1962, pp. 73 y ss.). No entraremos en el detalle de estos procedimientos, lo que excede por mucho el marco de este trabajo, sino que tomamos lo esencial de su movimiento conceptual tal como Vuillemin lo reconstruye. 
la materia) y metodológico (que consiste en su propia propuesta de abordaje, inversa pero complementaria con la de los algebristas previos). Así, pasa sigo la terminología de Vuillemin - de un abordaje a posteriori (histórico o 'apagógico') a uno a priori (directo), analizando mediante esta división el caso de la ecuación de tercer grado, de cuarto grado, y de grado superior a cuatro.

El método a posteriori (a través del cual Lagrange generaliza los procedimientos de sus predecesores) se basa en la búsqueda de una ecuación auxiliar o reducida, asociada a la ecuación propuesta (es decir, la ecuación de partida). La reducida es una ecuación adicional construida deliberadamente para simplificar la expresión de la propuesta mediante una (o una serie de) transformación(es) que permita(n) eliminar alguno de sus términos; esto se realiza sustituyendo la incógnita $x$ por una ecuación auxiliar cuyos resultados se suponen en relación racional con los de la propuesta, basándose en sus coeficientes. Obtenida la reducida, se operan sobre ella nuevas transformaciones para llegar a expresiones de un grado inferior (Vuillemin habla de "reducida rebajada" o "disminuida"), hasta poder obtener algunas de sus raíces mediante una fórmula general ya conocida. Se forman a continuación las expresiones del binomio asociado a la ecuación reducida disminuida, lo que permite operar con raíces cúbicas de la expresión para dar con la ecuación resolvente (la solución del problema), que expresa las soluciones de la reducida en función de las de la propuesta. Este es el procedimiento de los algebristas 'precríticos': se trata de un método que evoluciona por 'tanteo', partiendo de la ecuación dada y avanzando mediante la prueba de ecuaciones auxiliares construidas para sustituir la incógnita, yendo de la propuesta a la resolvente por medio de la reducida (Cf. Vuillemin, 1962, pp. 74-88).

Por otra parte, el método a priori o directo propuesto por Lagrange - que anuncia el 'período crítico' - traza un camino inverso (de la resolvente a la reducida, en vez de ir de la reducida a la resolvente), y se dirige a la cuestión de las condiciones de posibilidad de la resolución mediante la ecuación reducida. La reducida deja entonces de considerarse un mero momento auxiliar o accesorio - y por ende prescindible - en la búsqueda de la resolvente, y pasa a analizársela por sí misma en virtud de ciertas propiedades que la comparación con la resolvente revela en ella. Lagrange busca entonces comenzar por la resolvente (presenta un método de construcción general de lo que luego fue llamado resolvente de Lagrange), y mediante un análisis de sus relaciones con la reducida muestra las propiedades que convierten a esta última en un momento necesario del proceso de resolución. Este último punto supone - y en esto reside el genio de Lagrange - la intervención de un procedimiento de permutaciones en el grupo de raíces de la propuesta (todavía desconocidas), que 
permite demostrar que el grado de la reducida depende de las permutaciones posibles de dichas raíces en el contexto de una serie de funciones algebraicas asociadas a la resolvente. Esto permite determinar los rebajamientos de grado operables sobre la reducida, y construir las expresiones auxiliares necesarias para avanzar con la resolución. Esto supone un salto respecto al álgebra anterior pues añade a las operaciones algebraicas ya admitidas las operaciones con estructuras de grupos. ${ }^{11}$ Lagrange inaugura así una vía de fundamental importancia, pues gracias al análisis de los grupos de permutaciones de las raíces, el procedimiento de la búsqueda por 'tanteo' de los algebristas previos se reemplaza por un método racional o justificado, que muestra por qué (y en qué casos) aquel procedimiento funciona (es decir, en qué casos puede construirse una reducida eficiente). Lagrange concluye que la comparación con la resolvente le permite reducir ecuaciones generales de hasta cuarto grado, pero llegados al quinto esta función se vuelve inútil para dar con la reducida, porque en esos casos la resolvente es raíz de una ecuación de un grado superior a la propuesta, lo que impide, en general, la posibilidad de rebajar su grado (Cf. Vuillemin, 1962, pp. 78-80 y pp. 88-99).

La reflexión de Lagrange explora ambas perspectivas (a posteriori histórica y a priori directa) en torno a los tres casos de ecuaciones particulares estudiadas (tercer grado, cuarto grado, y superior a cuatro). Finalmente, en una cuarta (y última) sección, su artículo se desliga de la consideración de ecuaciones particulares, y se enfoca en el carácter general del método a priori directo: las estructuras de grupos de permutaciones de raíces, en las cuales se revelan los alcances y límites del proceso de construcción de resolventes. Allí se encuentra, según Vuillemin, un nuevo momento a priori, que constituye un momento metafísico:

[E]n las tres primeras secciones de su artículo, Lagrange construye las soluciones de la propuesta suponiendo dada la resolvente; en la cuarta sección, por el contrario, se pronuncia sobre la posibilidad misma de la construcción de la resolvente, cuando enuncia su teorema. Pero esta diferencia técnica parece ocultar una, más profunda, tocante a la Metafísica del método, de tal suerte que se podrá, por analogía, reencontrar

11 Un grupo es una estructura algebraica compuesta por un conjunto no vacío y una operación interna que vincula un par de sus elementos para obtener un tercero que forma parte del mismo conjunto. Un grupo es abeliano - en honor a Abel - cuando su operación interna satisface la propiedad conmutativa (de modo que el orden de los argumentos no incide en el resultado de la operación). Lagrange, y luego Abel, comienzan a usar estructuras de grupos conformadas por el conjunto de raíces de la ecuación propuesta y operaciones algebraicas que permitan discernir sus valores. Con Galois, la noción de grupo cobrará autonomía y mayor generalidad, revelando la razón de los éxitos y los fracasos en el dominio del cálculo de raíces de ecuaciones por radicales, e inaugurando a su vez a otros dominios que expanden notablemente el campo del álgebra. 
su articulación con otros dominios, sin relación de objeto con el problema del álgebra (Vuillemin, 1962, p. 100).

Este tercer momento del método, metafísico - y, por ende, también a priori, aunque en otro sentido que el segundo momento -, se vincula exclusivamente a las condiciones de posibilidad de construcción de la resolvente, "de la cual partía el primer momento a priori y en el cual finalizaba el momento apagógico" o a posteriori (Vuillemin, 1962, p. 102). Estos dos momentos, entonces, permanecían atados al contenido particular de las ecuaciones, y representan lo que Deleuze tildaba de "ilusión natural": ¿es esta fórmula resolvente adecuada para este tipo de ecuación propuesta? Por su parte, el método 'metafísico' de Lagrange, sin dejar de implicar un avance considerable en la historia del álgebra, cae en la ilusión filosófica: se pregunta por las condiciones de posibilidad de la construcción de las resolventes en sus aspectos formales. Al estar determinado por la construcción de la resolvente, este método 'metafísico' permanece adherido a los objetos individuales que han de servir de solución al problema (a saber, las ecuaciones). Si el método prelagrangiano era incapaz de elevarse sobre el contenido de los individuos algebraicos dados, el momento metafísico del método de Lagrange se enfoca en las condiciones formales de dichos individuos. En este punto (como indicaba el pasaje que venimos de citar) se da un salto respecto a los otros dos momentos que permite trazar analogías con "otros dominios", más allá del problema del álgebra. Vuillemin tiene en mente el paralelismo entre los métodos del álgebra con los de la filosofía crítica: a vuelta de página, traza una analogía entre el método de Lagrange y la filosofía de Fichte.

\section{4. Álgebra y filosofía crítica: el paralelismo Lagrange-Fichte}

En efecto, Vuillemin considera que los problemas algebraicos pueden cruzarse con los filosóficos, más allá de la aparente falta de relación histórica directa. Consciente de que esto puede resultar 'arbitrario', añade: "Pero si la resolución algebraica de las ecuaciones está ligada a las estructuras sin embargo lejanas de la Teoría de grupos, nada impide a priori ver, entre dominios más alejados aún, si no relaciones exactas de estructuras, al menos analogías en los procesos que ellas plantean" (Vuillemin, 1962, p. 102). Este pasaje es fundamental no solo para entender el espíritu con el cual el autor de $L a$ philosophie de l'algèbre cruza ambos dominios, sino también el que Deleuze ostenta al echar mano a herramientas matemáticas en el desarrollo de su propia 
filosofía (compartido también por Albert Lautman, otra influencia mayor de Deleuze en este ámbito). ${ }^{12}$

Solo a través del rodeo por la filosofía crítica podrá verse la potencia que Vuillemin (y, por extensión, Deleuze) encuentra en el método de Abel (que veremos en la próxima sección). Para esta filosofía, la ecuación a resolver (la propuesta) es el problema del origen de la validez objetiva o el sentimiento de necesidad que acompaña nuestras representaciones. Este problema no podía plantearse antes de Kant, pues se suponía dogmáticamente a la cosa en sí como produciendo directamente esas representaciones, o bien se negaba escépticamente toda validez objetiva en ellas. Sin embargo, según indica Vuillemin (Cf. 1962, p. 103), la revolución copernicana de Kant sostiene acríticamente la validez de las ciencias de su época (en particular la física y la matemática), que toma como hecho indubitable para establecer la doctrina de las facultades de la cual dependerá la validez objetiva buscada.

Vuillemin compara entonces a Kant con los algebristas previos a Lagrange, que operaban a posteriori, al tanteo y sin método, con una imagen preconcebida del tipo de solución al que se debía arribar. Su pensamiento puede dar con la verdad, pero no nos permite conocer sus fuentes, "porque se mantiene completamente en este juicio hipotético: si queremos concebir la posibilidad de la experiencia, entonces debemos poner tales combinaciones determinadas de elementos" (Vuillemin, 1962, p. 104). Lagrange, en cambio, sería equiparable a Fichte, ${ }^{13}$ quien cambia el modo de abordar la 'ecuación' del idealismo trascendental cuando sustituye la pregunta sobre las condiciones de posibilidad de la experiencia por la de las condiciones de posibilidad de la conciencia de sí, a partir de la cual se deduce la posibilidad de la experiencia (Cf. Vuillemin, 1962, p. 105). Esta sustitución - como el momento a priori del método de Lagrange - no modifica el resultado final de la crítica, pero le da un basamento más firme.

Al igual que en Lagrange, Vuillemin descubre en Fichte tres momentos. En un primer momento (que retoma los lineamientos principales de Kant, así

12 Dice Lautman: "Podrá parecer extraño a quienes están acostumbrados a separar las ciencias 'morales' de las ciencias 'exactas' el ver reunidas en un mismo trabajo reflexiones sobre Platón y Heidegger con observaciones sobre la ley de reciprocidad cuadrática o la repartición de los números primos. Quisiéramos haber mostrado que ese acercamiento de la metafísica y de las matemáticas no es contingente sino necesario." (Lautman, 2011, p. 333).

13 Las obras de Fichte en las que se apoya Vuillemin para su análisis son el Fundamento de toda la Doctrina de la Ciencia de 1794, el Compendio de lo propio de la Doctrina de la Ciencia en lo concerniente a la facultad teórica de 1795, y sus dos Introducciones a la Doctrina de la Ciencia, de 1797. El recorrido aquí planteado se basa exclusivamente sobre la interpretación de Vuillemin, dejando de lado la riqueza y complejidad propias del sistema fichteano. Para una lectura más cuidadosa sobre la relación de Deleuze con Fichte en torno al problema del dogmatismo y la filosofía crítica puede verse Ferreyra (2018). 
como Lagrange retomaba a los algebristas previos) una reflexión 'derivada' nos abstrae de la experiencia y nos revela la serie de nuestras representaciones como ligada esencialmente a nuestra conciencia. Allí, Fichte plantea su sustitución o su 'ecuación reducida', cuando pregunta por el modo en que el Yo se pone como determinado por el no-Yo (en vez de preguntar por la posibilidad de la experiencia). Este paso recuerda la posición de la reducida como momento simplificador y auxiliar, y la deducción que Fichte emprende desde ahí lo llevará, según Vuillemin, a un análogo de la resolvente de Lagrange. Se llega a esta al mostrar que la posibilidad de la experiencia depende de la experiencia de la actividad por la cual el Yo se pone como determinado por el no-Yo. Si bien 'materialmente' la reducida y la resolvente son semejantes (ambas remiten al juego entre el Yo y el no-Yo), ellas no lo son desde el punto de vista de la vivencia que despiertan en el filósofo que reflexiona: la resolvente transforma en experiencia vivida las representaciones previamente abstraídas, y las enlaza mediante la síntesis viviente de la imaginación productiva, donde el juego entre Yo y no-Yo genera la posibilidad de la consciencia de sí. Esto da lugar a una segunda serie o segundo momento del método: el de la reflexión originaria, que va en el sentido opuesto al de la primera. Aquí se parte de la resolvente (la síntesis viviente de la imaginación productiva) para mostrar la necesidad de la reducida (la oposición entre Yo y no-Yo).

En ambos casos, la deducción de la representación es la finalidad que guía el procedimiento. Pero la segunda serie da lugar, finalmente, a una nueva reflexión, o tercer momento del método: el punto de vista filosófico superior, por el cual se despierta a la conciencia vulgar de la pasividad, haciéndola reconocerse partícipe de la totalidad sintética de las actividades ocultas del Yo. Esto se da cuando el Yo reencuentra su decisión práctica como único medio para salir de las antinomias a las que lo conduce la razón teórica en la deducción de la representación; así, el punto de vista superior "reencuentra bajo forma de experiencia aquello que, antes de emprender la deducción de la conciencia real, ella se había asignado como principios abstractos de una conciencia meramente posible" (Vuillemin, 1962, p. 110). Esta tercera serie puede ser llamada (según Vuillemin) 'estructural', y posee su análogo en la estructura de grupo de permutaciones (tercer momento de Lagrange), puesto que, así como esta se elevaba a condición de posibilidad real de los dos momentos anteriores, aquí la pura actividad de autoposición del Yo es la condición real de la representación, que supone la posición del Yo y noYo como instancias recíprocamente determinadas, o divisibles, en la finitud efectiva de la experiencia. 
Vuillemin llama 'método genético' al procedimiento de los algebristas prelagrangianos y al de la filosofía kantiana, que buscan generar lo universal a partir de lo singular (sus intentos de dar una resolución general a sus problemas respectivos permanecen estrechamente condicionados por la imagen predeterminada de la peculiaridad de la solución). Este es el vicio del método genético, que recuerda a las 'ilusiones' deleuzianas.

En los dos sistemas, las dos primeras series ilustran un método que puede llamarse genético. La primera serie va de la propuesta a la resolvente mediante las soluciones; la segunda desciende de la resolvente a las soluciones que ella permite construir. En ambos casos, el método está ligado a su objeto: aquí la teoría de las ecuaciones, allí la teoría de la posibilidad de la representación. La tercera serie se libera al contrario de estos puntos de partida contingentes; ella es general y abstracta. [...] Puede verse entonces legítimamente a estas terceras series como ilustraciones del método estructural (Vuillemin, 1962, pp. 113-114).

El método estructural o general es la superación del vicio del método genético, que extrapolaba los elementos de partida a las condiciones generales que debían servirles de fundamento. Tanto en Lagrange como en Fichte, el tercer momento vislumbra la posibilidad de ese momento superador, capaz de revelar la estructura subyacente a los métodos previos de resolución de los problemas planteados. Ahora bien, si pueden considerarse estos puntos de vista como superadores, Vuillemin también señala que ambos arrastran, de un modo u otro, el resabio de elementos empíricos que mantienen sus métodos anclados en los datos que les sirven de punto de partida. En este sentido, ambos dan un paso para liberarse del método genético, pero vuelven a caer en él. Lagrange, por permanecer atado a la consideración de las ecuaciones individuales, lo cual le impide darle a la teoría de grupos su verdadera autonomía. Fichte, por recurrir a un 'choque' sensible que el Yo descubre como ocasión ininteligible de la que se desprenden sus síntesis efectivas, lo cual le impide pensar la intuición intelectual como realmente incondicionada (Cf. Vuillemin, 1962, pp. 117-118).

Todo esto remite, en términos deleuzianos, a la replicación de la ilusión natural en la ilusión filosófica: la natural afecta a los algebristas prelagrangianos que, como vimos, se lanzaban directamente a la búsqueda de la resolvente ante una ecuación dada; la filosófica afecta a Lagrange, que elabora un método para la construcción de la resolvente basado en la estructura del grupo de la ecuación, pero permanece adherido a la forma de la misma, lo que le impide ver en el grupo la apertura a un dominio superador a la consideración de las 
ecuaciones particulares. ${ }^{14}$ Por su parte, la ilusión natural afecta a la filosofía dogmática precrítica que consideraba a la representación como bien fundada en su inmediata relación con el objeto; la filosófica afecta a Kant (cuando supone la forma de la experiencia posible como un factum con el cual deben concordar los resultados formales de la tarea crítica) e incluso a Fichte (según Vuillemin, al suponer un choque ininteligible como factum más allá de toda posibilidad de deducción). Así, tanto Lagrange como Kant y Fichte caen en el círculo vicioso. El universal al que llegan conserva el recuerdo de su origen contingente: "todo método genético es un empirismo larvado" (Vuillemin, 1962, p. 118). La auténtica revolución crítica en el álgebra, que consiste en el despliegue completo del método estructural o 'general', estará dada por el trabajo de Abel en ecuaciones quínticas.

\section{Abel y la revolución crítica en álgebra}

Abel es recordado principalmente por su trabajo en integrales de funciones elípticas y por haber probado la imposibilidad de una fórmula general para resolver por radicales el polinomio de grado cinco o mayor (se realiza esto previamente a la teoría de grupos de Galois, que no solo permitió establecer un método alternativo de resolución, sino que inauguró un nuevo campo para el álgebra). Su demostración es conocida hoy como teorema Abel-Ruffini, pues retoma y completa un procedimiento desarrollado por el matemático italiano (algunos de cuyos pasos no estaban debidamente probados). De acuerdo con Vuillemin, el método en sí mismo no supone un avance radical desde el punto de vista de la técnica algebraica (cosa que sí introduce Galois), pero sin embargo la exposición del método reviste un interés filosófico fundamental. En su desarrollo, Abel presenta una perspectiva que rompe con el vicio que Vuillemin detectaba todavía en Lagrange: su carácter 'genético' que, por permanecer en la consideración de las ecuaciones particulares a las cuales debía adaptarse, no llegaba a vislumbrar el problema desde su planteo más general o estructural. La ruptura de Abel pasa precisamente por el carácter general e indirecto del planteo que conduce a su demostración (Cf. Vuillemin, 1962, p. 207). Este método general, que supera al genético - invirtiendo así el matiz axiológico que Deleuze otorga a estos términos $-{ }^{15}$ consiste en analizar

14 De acuerdo con Vuillemin (Cf. 1962, p. 117), este mismo problema alcanza a las intervenciones de Lagrange en teoría de funciones y en mecánica analítica; el método estructural será definitivamente realizado por Abel y Galois, Cantor y Grassmann.

15 En efecto, a lo largo de Diferencia y repetición pueden encontrarse numerosas referencias que van en contra de las 'generalidades abstractas' del mundo de la representación, y en favor del carácter 'genético' de la filosofía 
el problema planteándolo en su mayor generalidad formal. Abel indica que los métodos de sus predecesores eran incapaces de conducir a buen puerto, excepto en el caso en que las ecuaciones abordadas fueran de hecho resolubles - cosa que ellos daban acríticamente por descontada. El propio carácter de resolubilidad de la ecuación propuesta no era en sí mismo una cuestión que se considerara digna de evaluación, sino que se procedía inmediatamente a intentar resolverla.

En efecto, se proponía resolver las ecuaciones sin saber si esto era posible. En este caso, se podía llegar a la resolución, aunque esto no era del todo certero; pero si por desgracia la resolución era imposible, se hubiera podido buscarla una eternidad, sin encontrarla. Para llegar infaliblemente a algo en esta materia, hay que dar al problema una forma tal que sea siempre posible resolverlo, cosa que siempre es posible para cualquier problema. En lugar de preguntar por una relación que no se sabe si existe o no, hay que preguntar si una relación tal es en efecto posible (Abel, 1828, p. 85). ${ }^{16}$

Así comienza Abel el tratado donde presenta su demostración de imposibilidad: denunciando la falta de análisis sobre las condiciones del problema a abordar, por la que pecaron sus antecesores. Dada la ecuación propuesta, todos ellos suponían de antemano que era resoluble y procedían a elaborar métodos para resolverla, pero ni la cuestión de si la ecuación propuesta era efectivamente resoluble, ni la naturaleza misma de esa resolubilidad eran puestas en cuestión. En efecto, el método seguido por Lagrange y los algebristas previos solo podía conducir a conclusiones certeras en el caso de que la ecuación propuesta fuera resoluble por radicales; ellos suponían que la relación buscada entre raíces y coeficientes de la ecuación existía necesariamente, y que era expresable mediante las herramientas algebraicas ya disponibles. Abel da un paso atrás, mostrando la necesidad de explicitar en qué consiste dicha relación y en qué casos ella es posible.

Por eso, la exposición de Abel consiste en definir la forma más general de una ecuación algebraica, pero indicando luego que esta forma debe subordinarse a la forma del problema: para eso, hay que definir las condiciones que debe satisfacer una ecuación algebraicamente resoluble. Abel nota que deben existir relaciones entre las raíces de la ecuación tales que cada una debe poder ser

de la diferencia. En el presente contexto, el carácter 'general' del método de Abel se corresponde con lo que Deleuze considerará su 'estructuralismo', única vía para alcanzar un auténtico carácter genético desde el aspecto virtual del problema. En cambio, lo que Vuillemin Ilama 'método genético' se asocia justamente a la generación de generalidades vacías de la representación, o su abstracción a partir de caracteres individuales empíricos para formar un falso universal.

16 Deleuze (Cf. 1968, p. 233) menciona en nota a pie de página, junto con el libro de Vuillemin, este artículo de Abel; las cursivas en "forma" son nuestras. 
expresada por funciones racionales de las raíces restantes. A continuación, apela al método de las sustituciones (al que se dirigían precipitadamente los algebristas precríticos), y da un criterio general sobre el número de valores diferentes que puede tomar una función de grado $n$, que no puede reducirse más allá del primer número primo que sobrepase a $n$, exceptuando 1 y 2 (estableciendo así las condiciones en las que una ecuación reducida es posible o no). Demuestra a partir de ahí la imposibilidad de la resolvente general de la ecuación de quinto grado. Durante todos los pasos del desarrollo, Abel procura plantear el problema de la forma más general posible, sin concentrarse en ejemplos o casos de ecuaciones particulares, sino en las relaciones estructurales implícita o inconscientemente supuestas en los abordajes previos.

En resumen, se analiza en su forma más general una relación o un conjunto de relaciones; se analiza en su forma más general la clase de seres a los cuales es posible atribuir esta relación; se analizan, en fin, las compatibilidades e incompatibilidades entre estos seres y estas relaciones. Como señalamos, más allá del análisis técnico, interesa la renovación filosófica que Vuillemin desprende de la exposición de este método general, en oposición al método genético. En la teoría de las ecuaciones, el problema del polinomio de quinto grado permanecía irresuelto pues los procedimientos utilizados en los grados inferiores ocultaban la razón de fondo que aseguraba el éxito en la resolución - a saber, el hecho de que cada raíz de una ecuación algebraicamente resoluble se compone de funciones algebraicas expresables a su vez por funciones racionales de las raíces restantes. Dado que esos procedimientos funcionaban a la perfección con aquellos 'individuos algebraicos' (las ecuaciones de segundo, tercer y cuarto grado), se abordaban también las quínticas bajo la misma lente. Pero estas últimas se resistían sistemáticamente a un tratamiento adecuado mediante esos procedimientos, en tanto suponían la apertura a un dominio que no solo excedía el poder expresivo de los mismos, sino que además mostraba cuándo, cómo y porqué ellos eran exitosos. Era necesario replantear el problema en toda su generalidad formal o estructural para que este aspecto, previamente oculto, se develara como lo esencial del asunto.

Por este carácter general del método, la pureza formal de la expresión deja de ser un mero accidente (una cuestión de prolijidad o elegancia) y deviene esencial a la demostración - esta es la marca dejada por Abel en la historia de la matemática, que conduce al estructuralismo matemático; de hecho, David Hilbert, fundador del formalismo (e inspirador del estructuralismo axiomático de Lautman), dio la mayor importancia al trabajo de Abel (Cf. Vuillemin, 1962, pp. 214-215). Es entonces preciso remarcar que la 'generalidad' del método de Abel no consiste en una abstracción por generalización de casos 
particulares, sino en una formalización de las relaciones comunes a un cierto tipo general de individuos, definidos a su vez por dichas relaciones, de modo tal que las relaciones toman una autonomía relativa respecto a los individuos. Vuillemin ve aquí el surgimiento, en álgebra, del método general o estructural que Lagrange solo alcanzó a indicar de modo tangencial e inacabado:

\begin{abstract}
Porque tomaba su punto de partida en 'naturalezas simples' individuales y pasaba por analogía de lo especial a lo general, el método genético no podía nunca estar absolutamente seguro de no mezclar lo accesorio a lo esencial. En todo momento, las propiedades que definían a los individuos sobre los cuales se proponía reflexionar venían a mezclarse inextricablemente con las propiedades de las estructuras, enteramente independientes de los individuos que las ilustraban, y de las cuales dependía en realidad la solución del problema planteado. [...] En consecuencia, para dar al método genético su estilo verdaderamente crítico, hay que tomar otro método, y analizar las estructuras de las que dependen las clases definidas de problemas de manera relativamente independiente de los individuos empíricos a los que se aplican (Vuillemin, 1962, pp. 215-216).
\end{abstract}

Al igual que en Lautman, el auténtico aspecto genético va de la mano con el aspecto estructural: tras la crítica, ellos no funcionan como métodos aislados. Así lo afirma explícitamente Deleuze: "En conformidad con los trabajos de Lautman y Vuillemin sobre las matemáticas, el 'estructuralismo' nos parece el único medio por el cual un método genético puede realizar sus ambiciones" (Deleuze, 1968, p. 237). ${ }^{17}$ Estas 'ambiciones' de dar con lo universal se ven frustradas siempre que ese universal sea concebido a partir de los caracteres de los individuos sobre los cuales debe valer como tal. En lugar de buscar qué fórmula individual podía servir como resolvente general (como hacían los algebristas precríticos sumidos en la ilusión natural), o bien qué condiciones debía satisfacer tal o cual fórmula individual para constituirse como tal resolvente (como hacía Lagrange, preso de la ilusión filosófica y su círculo vicioso), Abel busca definir el dominio de condiciones generales o de relaciones puramente formales que caracterizan el vínculo entre los individuos en cuestión (la ecuación propuesta, la reducida y la resolvente). Esto implica precisamente un desplazamiento respecto al modo de plantear el problema, que exige ir más allá del ámbito de las relaciones concretas entre esos individuos, hacia un dominio de relaciones puramente formales, a la vez determinante y relativamente independiente de ellos. Este dominio era invisibilizado al enfocar que debe buscarse el sentido profundo de las páginas más estructuralistas de Diferencia y repetición. 
el problema exclusivamente desde los caracteres manifiestos de los individuos que lo suponían.

\section{Palabras finales: la filosofía trascendental deleuziana como una nueva Crítica}

El vicio del método genético era denunciado por Vuillemin en matemática (con los prelagrangianos y Lagrange) y también en filosofía (con Kant y Fichte), lo cual sugería la necesidad de realizar también aquí una revolución del tipo de la abeliana. Las páginas en las que La philosophie de l'algèbre reflexiona sobre las implicancias filosóficas del método abeliano tienen sorprendentes resonancias con la teoría deleuziana de los problemas y de las facultades; por ejemplo, cuando afirma: "toda la filosofía clásica permanece atada al método genético. La génesis, a fin de cuentas, llama siempre a la teología que ella quería rechazar, porque los límites que pretende asignarle a las facultades del conocimiento no nacen interiormente de la estructura de las facultades, sino que les vienen de afuera, por los objetos individuales a los que se aplican" (Vuillemin, 1962, p. 217). Se trata del círculo vicioso que, territorializado en el proyecto de una filosofía de la diferencia, Deleuze denuncia en la teoría de los problemas: concebir lo trascendental (lo problemático) a imagen y semejanza de lo empírico (los casos de solución).

Según Vuillemin, esta extrapolación ilegítima ocurría - en el campo de la filosofía trascendental - con la noción kantiana de experiencia posible y con el 'choque' fichteano, instancias que les impedían resolver satisfactoriamente su 'ecuación propuesta' - es decir, la deducción, a partir de la pura razón, del carácter de objetividad atribuido a nuestras representaciones. Vuillemin denuncia en el método genético un paso ilegítimo que hipostasia en el ámbito trascendental ciertas propiedades de esas representaciones que ese ámbito debía justificar. Por eso, luego de exponer las características algebraicas del método general de Abel, esboza la idea de una crítica general de la razón pura (Cf. Vuillemin, 1962, pp. 218-221). Recupera para esto el ejemplo de la refutación kantiana del argumento ontológico, y afirma la necesidad de reformularlo en términos de un análisis intrínseco al concepto de infinito. Dicho análisis debe sostener la recusación kantiana de la metafísica dogmática, poniendo límites al uso de la razón, pero esos límites no pueden venir de los objetos de una facultad exterior a la razón misma, como la intuición sensible, pues haciendo esto solo se refuerza el carácter exterior y contingente del método. Así, se malogra la crítica inmanente de la razón por sí misma, que debía caracterizar la filosofía trascendental. 
La cuestión de una crítica general de la razón pura queda abierta en Vuillemin - no hay aún, para él, un 'Abel de la filosofía' (así como había un Fichte en analogía con Lagrange). Deleuze parece adjudicarse a sí mismo este lugar en el esquema de Vuillemin, cuando desarrolla su teoría de los problemas dialécticos y recupera a Abel para mostrar la ruptura del círculo vicioso que ligaba las ilusiones en torno a la teoría de los problemas: "si un círculo tal ha sido roto, es ante todo por el matemático Abel; es él quien elabora todo un método siguiendo el cual la resolubilidad debe desprenderse de la forma del problema. Se ha podido afirmar que Abel inauguraba así una nueva Crítica de la razón pura, y superaba precisamente el extrinsequismo kantiano" (Deleuze, 1968 , p. 233). Únicamente en el giro crítico que supone el método general abeliano es posible sentar las condiciones de una auténtica génesis, una génesis situada en los problemas mismos, lo cual implicará para Deleuze la posibilidad de hacer frente a la ecuación de la filosofía trascendental: dar cuenta del mundo de la representación sin que sus características (la identidad, la semejanza, la oposición y la analogía) se inmiscuyan en el discurso filosófico.

Desarrollar este proyecto en toda su generalidad motivará la creación de la batería de conceptos desplegada en los últimos capítulos de Diferencia y repetición. Allí, las puras relaciones independientes de todo carácter individual, definidas como relaciones diferenciales, caracterizan el plano virtual del problema. Este plano se acoplará al de la intensidad (desarrollado en el capítulo V de Diferencia y repetición) formando la 'ecuación reducida' del problema de la filosofía crítica (que Fichte planteaba en términos de oposición entre Yo y no-Yo). Abel y Vuillemin concurren como fuentes que proveen a Deleuze de herramientas para pensar el pensamiento, liberado de la imagen dogmática que le imponía límites externos ilegítimamente extrapolados del ejercicio empírico de las facultades y sus objetos. El problema, así definido, es caracterizado como una fuente de puras relaciones, determinaciones virtuales o preindividuales que constituyen la potencia de un campo de individuación intensivo, llevadas a un espacio-tiempo individual por un dinamismo espacio-temporal correspondiente a ese campo (Cf. Deleuze, 1968, pp. 276-285 y 315-318). Los procesos propios de este campo de individuación intensiva, sin embargo, exceden con mucho los objetivos de este trabajo, que se propuso especificar la distinción entre el plano problemático virtual o preindividual y el de los individuos constituidos. ${ }^{18}$

18 La relación entre las instancias de lo virtual, lo actual y lo intensivo es de hecho uno de los principales puntos de debate en torno a la ontología deleuziana de Diferencia y repetición, y no hemos intentado aquí sino presentar una reflexión localizada exclusivamente en el aspecto virtual. Para un recorrido por esta cuestión puede verse Clisby (2016). 
En conclusión, y para resumir, el método general de Abel ilustra los caracteres fundamentales de la teoría deleuziana de los problemas virtuales: la heterogeneidad del problema respecto a la solución (independencia de lo empírico respecto a lo trascendental); la dependencia relativa de la solución respecto al problema; la autodeterminación inmanente del problema en el proceso de resolución; la potencia del problema para abrir los dominios de soluciones preexistentes a la génesis de otros dominios epistemológicos o simbólicos (así como, en álgebra, la teoría de las ecuaciones da lugar a la teoría de grupos); el carácter estructural del problema como complejo de puras relaciones $-\mathrm{y}$ relaciones entre relaciones - sin consideración a los términos actuales o individuos que las instancian.

\section{Bibliografía}

ABADI, D. "El aporte algebraico de Galois a la teoría deleuziana de los problemas". In: Ágora: Papeles de filosofía, Vol. 34, Nr. 2, 2015, pp. 25-43. https://doi.org/10.15304/ ag.34.2.2108.

ABEL, N. H. (1828) "Sur la résolution algébrique des équations". In : Euvres Complètes de N. H. Abel. T. II. Christiania: Grundähl, 1839.

CANAVERA, J. "Notas sobre la crítica de la imagen dogmática en la obra de Gilles Deleuze". Revista de Filosofia, Vol. 40, Nr. 2, 2015, pp. 83-108. http://dx.doi.org/10.5209/ rev_RESF.2015.v40.n2.50057.

CLISBY, D., “¿El dualismo secreto de Deleuze? Versiones en disputa sobre la relación entre lo virtual y lo actual”. Traducción española de Pablo Pachilla. Ideas. Revista de filosofía moderna y contemporánea, Nr. 4, 2016, pp. 120-148. http://revistaideas.com. ar/ideas04nota05/.

DELEUZE, G. “Différence et répétition”. París: PUF, 1968.

. "Exasperación de la filosofía: El Leibniz de Deleuze". Traducción española del equipo editorial Cactus. Buenos Aires: Cactus, 2006.

DELEUZE, G. y GUATTARI, F. “Mille Plateaux”. París: Les Éditions de Minuit, 1980. DOSSE, F. "Gilles Deleuze et Félix Guattari. Biographie croisée”. París: Editions La Découverte, 2007.

DUFFY, S. "Deleuze and the History of Mathematics: In Defense of the New". Londres/ Nueva York: Bloomsbury, 2013.

FERREYRA, J. "Fichte o Deleuze, ¿quién es el dogmático?”. Valenciana, Vol. 11, Nr. 21, ene./jun. 2018, pp. 257-288. http://dx.doi.org/10.15174/rv.v0i21.326.

FERREYRA, J. y SOICH, M. (eds.) "Deleuze y las fuentes de su filosofía". Buenos Aires: La Almohada, 2014.

KLINE, M. (1972) "Historia del pensamiento matemático desde la antigüedad hasta nuestros días". Traducción española de Mariano Martínez, Juan Tarrés y Alfonso Casal. Madrid: Alianza, 1992. 
KRETSCHEL, V. "Lautman y Deleuze: Idea, problema, estructura y realidad". In: Ferreyra, J., y Soich, M. (eds.), 2014, pp. 28-36.

LAGRANGE, J.-L. (1771). "Réflexions sur la résolution algébrique des équations". In: Euvres complètes de Lagrange. T. III. París: Gauthier-Villars, 1869, pp. 205-421. LAUTMAN, A. (1771). "Ensayos sobre la dialéctica, estructura y unidad de las matemáticas modernas". Traducción española de Fernando Zalamea. Bogotá: Universidad Nacional de Colombia, 2011.

SANTAYA, G., "El procedimiento de adjunción de Évariste Galois y su presencia en la ontología deleuziana". In: J. Ferreyra y M. Soich (eds.), 2014, pp. 37-47.

. "El cálculo trascendental. Gilles Deleuze y el cálculo diferencial: ontología e historia". Buenos Aires: RAGIF Ediciones, 2017.

VUILLEMIN, J. "La philosophie de l'algèbre”. París: PUF, 1962. 